\title{
Temperature Sensors and Controls for the Duke FEL Storage Ring
}

\author{
P.H. Morcombe, Y. Wu, Robert McGehee* \\ Duke University, Free Electron Laser Laboratory, P.O. Box 90319, Durham, NC 27708
}

\section{Abstract}

This paper describes a prototype system used for sensing and control functions associated with the Duke University $1 \mathrm{GeV}$ electron storage ring. Low cost industrial control systems have been used. The system is capable of handling at least four hundred devices.

\section{INTRODUCTION}

The Duke storage ring uses deionized water to cool most of its high power devices. Initially, the water cooling systems gave very little trouble but over time the incidence of blocked cooling lines increased. Adding water filters improved the situation but did not entirely eliminate blockages. All critical items such as magnet coils are fitted with "Klixons" interlocked with the power supplies. While the Klixons prevent devices from being damaged through over heating, they also cause the stored electron beam to be lost without warning. As the blockage of the cooling system pipes is usually a gradual process, an early warning system capable of detecting partial blockages is highly desirable.

\section{SYSTEM DESIGN}

Given our very tight budget, approaches based on measuring the water flow directly were too expensive. Indirect approaches based on temperature measurement opened up a wide range of excellent low cost equipment and provided some side benefits.

The Dallas Semiconductors DS 1820 Digital Thermometer was chosen to perform the sensing task. This device measures temperature from -55 to $+125{ }^{\circ} \mathrm{C}$. The DS 1820 contains all the electronics needed to communicate over a Dallas Semiconductor MicroLAN. Each DS 1820 has its own unique address and converts temperatures into digital words that are transmitted over the MicroLAN under control of a server. The DS 1820 extracts the power it needs from the MicroLAN. The system configuration is shown in Fig. 1:

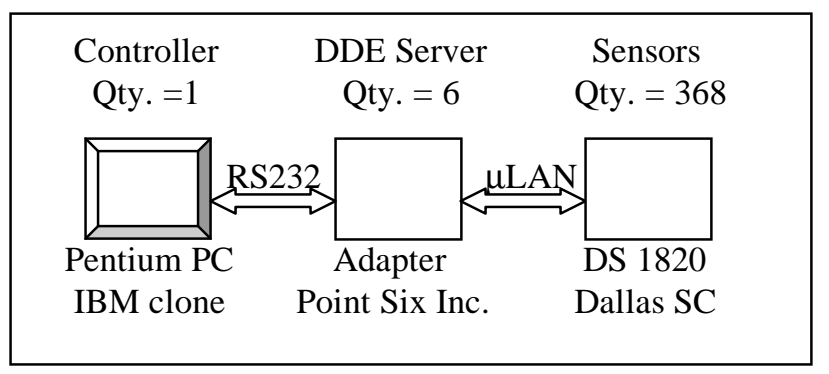

Fig 1. System configuration
The controller is a Pentium PC running under Windows 95. The data collection and display is handled by Excel 97 using DDE (Dynamic Data Exchange) to communicate with the six MicroLAN servers. The system programming consists of Excel macros written using Microsoft's VBA (Visual Basic for Applications) development tools and libraries.

Each MicroLAN server consists of a hardware adapter and software that runs on the PC. These items were provided by Point Six Inc. of Lexington, KY.

The system displays current temperature in bar chart form and logged data in line graph form. Devices that are over heating are collected in an alarm display. For easier viewing, the devices have been divided up into groups of 44 , selected by means of pull down menus. It is also possible to customise the display by picking devices from a list. Anyone familiar with Microsoft Excel can learn to use the system in half an hour.

\section{OPERATING EXPERIENCE}

The system came into operation in mid August 1998 with 256 temperature sensors. The system worked well, so more devices were added:

\section{Devices}

Quadrupole magnets

Sensors

Dipole magnets

256

RF cavity

RF amplifier system

TOTAL

86

18

8

With 368 sensors, we are approaching the limit of the system as originally designed. However, improvements to the server hardware and software have the potential to increase the system capacity by a factor of five or more.

The system has enabled us to reduce the incidence of blockages to a very low level. A weekly review of temperatures is followed by preventative maintenance as necessary. The primary objective for installing the system has therefore been accomplished.

We are finding additional uses for low cost temperature sensing. For example, we are able to track power dissipation in our high power RF amplifier, its associated three port circulator and in all 17 water cooling loops on the ring accelerator cavity. We expect to be able to detect problems in these systems before catastrophic failure occurs.

\footnotetext{
" Currently an undergraduate at Williams University
} 
The use of shrink wrapped software essentially eliminated the need for professional programmers. This helped to speed up the system installation and subsequent system expansions. The cost of the entire system including the computer, adapters, cables, terminal blocks, sensors and software averaged \$24 per temperature sensor.

\section{CONCLUSIONS}

While the system is not suited to the high speed or high precision functions that our main EPICS control system performs, it has the potential to perform low speed sensing and control functions at a very low cost.

\section{ACKNOWLEDGEMENTS}

The authors thank all members of the Duke FEL Laboratory, especially Hank Goehring and Jim McDuff who installed the hardware and the DDE software links. The work was supported by the U.S. Office of Naval Research under Grant Number N00014-94-1-0818.

\section{REFERENCES}

[1] Dallas Semiconductor, “Application notes \#106 \& \#108”, (1995)

[2] Dallas Semiconductor, "Spec. DS 1820 Digital Thermometer", (1995)

[3] Point Six Inc., "Spec. HA-4 Isolated Host Adapter”, (1998)

[4] Point Six Inc., “DDE server version 1.6h”, (1998) 